\title{
The Nobel Prize in Physiology or Medicine 2021 was Awarded Jointly to Researchers-A 'touchy' story.....
}

The Nobel Prize in Physiology or Medicine 2021 was awarded jointly to researchers, David Julius,66, and Ardem Patapoutian, 54, from the University of California, San Francisco and Scripps Research in La Jolla, California, respectively recognising their extraordinary work in identifying and understanding the mechanism through which our body perceives temperature and pressure.

As Professor Abdel El Manira, member of the the Nobel Assembly said, "This discovery has profoundly changed our view of how we sense the world around us".

Our ability to sense heat, cold and touch determines our interaction with the world around us and is essential for survival. We take these sensations for granted in our daily lives. But how are the nerve impulses for temperature and pressure perception initiated? This question has been answered by this year's Nobel Prize laureates.

David Julius at the University of California, San Francisco, USA, saw the possibility for major advances by analyzing how the chemical compound capsaicin causes the burning sensation we feel when we come into contact with chili peppers. Capsaicin was already known to activate nerve cells causing pain sensations, but how this chemical actually exerted this function was an unsolved riddle. Julius and his co-workers created a library of millions of DNA fragments corresponding to genes that are expressed in the sensory neurons which can react to pain, heat, and touch. Julius and colleagues hypothesized that the library would include a DNA fragment encoding the protein capable of reacting to capsaicin. They expressed individual genes from this collection in cultured cells that normally do not react to capsaicin. After a laborious search, a single gene was identified that was able to make cells capsaicin sensitive. Further experiments revealed that the identified gene encoded a novel ion channel protein and this newly discovered capsaicin receptor was later named TRPV1. When Julius investigated the protein's ability to respond to heat, he realized that he had discovered a heat-sensing receptor that is activated at temperatures perceived as painful. David Julius' discovery of TRPV1 was the breakthrough that allowed to understand how differences in temperature can induce electrical signals in the nervous system.

Patapoutian and his collaborators on their path to identify the mechanism of pressure perception, first identified a cell line that gave off a measurable electric signal when individual cells were poked with a micropipette. It was assumed that the receptor activated by mechanical force is an ion channel and in a next step 72 candidate genes encoding possible receptors were identified. These genes were inactivated one by one to discover the gene responsible for mechanosensitivity in the studied cells. Patapoutian and his co-workers finally succeeded in identifying a single gene whose silencing rendered the cells insensitive to poking with the micropipette after a tedious search. A new and entirely unknown mechanosensitive ion channel had been discovered and was given the name Piezo1, after the Greek word for pressure. Through its similarity to Piezo1, a second gene was discovered and named Piezo2. Sensory neurons were found to express high levels of Piezo2 and further studies firmly established that Piezo1 and Piezo2 are ion channels that are directly activated by the exertion of pressure on cell membranes. Piezo1 and Piezo2 channels have further been shown to regulate additional important physiological processes including blood pressure, respiration and urinary bladder control.

The ground-breaking discoveries of the TRPV1, TRPM8 and Piezo channels by this year's Nobel Prize laureates have allowed us to understand how heat, cold and mechanical force can initiate the nerve impulses that allow us to perceive and adapt to the world around us. This knowledge is being used to develop treatments for a wide range of disease conditions, including chronic pain.

Time to applaud their contribution in explaining the complexity behind simple physiological functions in a marvel called life.....

Binita Goswami Associate Editor

Indian Journal of Medical Biochemistry romu11@rediffmail.combinita.dr@gmail.com 